\title{
Erratum to: Isoform specific phosphorylation of p53 by protein kinase CK1
}

\author{
Andrea Venerando - Oriano Marin • \\ Giorgio Cozza • Victor H. Bustos - Stefania Sarno • \\ Lorenzo Alberto Pinna
}

Published online: 5 January 2011

(C) Springer Basel AG 2010

Erratum to: Cell. Mol. Life Sci. (2010) 67:1105-1118

DOI 10.1007/s00018-009-0236-7

It has come to our attention that the following acknowledgment is missing in the article:

\section{Acknowledgments}

This work was supported by the European Commission (PROKINASERESEARCH 503467) and by the Associazione Italiana Ricerca sul Cancro (AIRC).

The online version of the original article can be found under doi:10.1007/s00018-009-0236-7.

A. Venerando · O. Marin · V. H. Bustos · S. Sarno · L. A. Pinna

Venetian Institute of Molecular Medicine (VIMM),

Via G. Orus, 2, 35129 Padova, Italy
A. Venerando - O. Marin - G. Cozza - S. Sarno ·
L. A. Pinna $(\bowtie)$
Department of Biological Chemistry, University of Padova,
Viale G. Colombo, 3, 35131 Padova, Italy
e-mail: lorenzo.pinna@unipd.it
Present Address:
V. H. Bustos
Laboratory of Molecular and Cellular Neuroscience,
The Rockefeller University, New York, NY 10065, USA 